\title{
Ameloblastoma- A Case Report
}

\author{
Dr. Anish Gupta, Dr. Shweta Gandhi \\ (Dept of Oral Pathology \& Microbiology, People's Dental Academy, People's University, India) \\ (Dept of Oral Pathology \& Microbiology, Vaidik Dental College \& Research Centre, India)
}

\begin{abstract}
This article highlights features of ameloblastoma which could be mistaken for a malignant tumor. Ameloblastoma is usually multilocular but may also present itself as unilocular radiolucency. Ameloblastomas are nototrious for their local invasiveness and their tendency to recur. It shows an aggressive behavior leaving surgical resection as one of the most practical approach towards treatment. Also with proper and calculated treatment options, reasonably fair results can be expected in terms of esthetics and function. We present an extensive case of plexiform ameloblastoma of the mandible in a female patient.
\end{abstract}

Keywords: Ameloblastoma, Plexiform ameloblastoma

\section{Introduction}

According to the WHO 1992, ameloblastoma is a benign but locally invasive polymorphic neoplasm consisting of proliferating odontogenic epithelium, which usually has a follicular or plexiform pattern, lying in a fibrous stroma. ${ }^{[1]}$ It represents $1 \%$ of all tumors and cysts that involve the maxillomandibular area and about $10 \%$ of the odontogenic tumors. ${ }^{[2]}$ The first description of Ameloblastoma was made by Cusack in 1827 . The term Ameloblastoma was given by Churchill in 1930. ${ }^{[3]}$ It is a very aggressive neoplasm by nature that arises from the remnants of the dental lamina and dental organ with patients presenting late in life after the tumor has reached an enormous size leading to facial disfigurement. ${ }^{[2]}$ Here we report a case of Plexiform ameloblastoma of mandible.

\section{Case Report}

A patient had reported in the department of Oral Medicine and Radiology at Vaidik Dental College \& Research Centre, Daman with a complaint of swelling on left side of lower jaw since 9 months. Patient gave $\mathrm{H} / \mathrm{O}$ swelling which was smaller in size initially, and reached present size rapidly with no H/O discharge and pain. Patient also gave H/O mal-positioning of teeth in lower front region. On inspection, Extra-oral swelling was seen on left side of mandible extending from mid-line upto angle of mandible medio-laterally and from the ala-tragus line to $1 \mathrm{~cm}$ below the lower border of mandible, size approximately $12 \times 7 \mathrm{cms}$, roughly oval shaped, overlying skin was normal and no visible discharge was seen [Figure 1]. The swelling was non-tender, hard in consistency and no localized rise of temperature. On Intra-oral examination, swelling was seen extending from lower right canine region to the left side of retromolar region laterally and from the ridge to vestibule superiorinferiorly, size being $10 X 7 \mathrm{cms}$ approximately and roughly oval shaped [Figure 2]. The surface was rough with no visible discharge seen. On palpation the swelling was firm to hard in consistency, non-tender. The panoramic radiograph showed well defined radiolucent shadow in the mandibular anterior region extending from the mesial surface of lower right first molar to the distal surface of lower left third molar medio-laterally, and from the interdental region of lower anterior to $1 \mathrm{~cm}$ below the lower border of mandible superior-inferiorly, roughly oval shaped size approximately $10 \times 6 \mathrm{~cm}$. The borders were well corticated, internal structure showed homogeneously radiolucent region with few septae. The surrounding structure showed resorption of the roots of involved teeth. Also extension of cortical plates were seen in the symphysis region.

\section{Discussion:}

Ameloblastoma is characterized by the proliferation of epithelial cells arranged on a stroma of conjunctive vascular tissue in locally invading structures that resemble the enamel organ at different stages of differentiation. ${ }^{[4]}$ Diverse histological patterns have been described in the literature and include follicular, plexiform, acanthomatous, papilliferous-keratotic, desmoplastic, granular, vascular and those with dentinoid induction. It is generally accepted that there is no relationship between the individual patterns and the behavior of the tumor or its prognosis. ${ }^{[7]}$ Approximately $80 \%$ of ameloblastomas occur in the mandible, usually in the posterior region. ${ }^{[5]}$ A notable exception is African Blacks, in whom any region of the mandible may be involved. ${ }^{[6]}$ Patients may present with a slow-growing mass, malocclusion, loose teeth, or more rarely paresthesia and pain; however, many lesions are detected incidentally on radiographic studies in asymptomatic patients. ${ }^{[5]}$ The tumor found in our patient was an ameloblastoma of the plexiform type. The term plexiform refers to the appearance of anastomosing islands of odontogenic epithelium in contrast to a follicular pattern. ${ }^{[4]}$ Histopathological features in our case showed anastomosing sheets and cords of odontogenic epithelium. The 
epithelium displayed a stellate, reticulum-type appearance, arranged as a tangled network of anastomosing strands, enclosing cysts of various sizes. Radiographically, ameloblastoma of the mandible can mimic other tumors of the mandible, such as, the odontogenic keratocyst, aneurysmal bone cyst, fibrosarcoma, or a giant cell tumor. So, its final diagnosis can only be confirmed with a histopathological examination.

The mainstay of treatment is surgery, with wide resection recommended due to the high recurrence rate of solid/multicystic ameloblastomas. The recurrence rate after resection is $13-15 \%$, as opposed to $90-100 \%$ after curettage. ${ }^{[8]}$ Many authors recommend a margin of $1.5-2 \mathrm{~cm}$ beyond the radiological limit to ensure all microcysts are removed. ${ }^{[9]}$ Several studies suggest treatment as an important prognostic factor, specifically implicating undertreatment as a cause of recurrence. ${ }^{[8]}$ While not a first line treatment, radiotherapy should be considered for patients with positive margins who are not amenable to re-excision. ${ }^{[5]}$ Follow-up is essential as most recurrences present within the first 5 years; however, some have been observed more than 10 years after initial treatment. ${ }^{[9]}$

\section{Conclusion:}

In conclusion, although ameloblastoma is one of the most common odontogenic tumors. A high index of suspicion of ameloblastoma will help triage the patients for further appropriate management.

\section{References:}

[1]. Sharma S, Goyal D, Ray A, Gupta N. Ameloblastoma in children: Should we be radical?. J Indian Soc Pedod Prev Dent 2011;29:74-8 (PROPER)

[2]. Chauhan D S and Guruprasad Y. Plexiform Ameloblastoma of the Mandible. J Clin Imaging Sci. 2011;1:61.

[3]. Amzerin M, Fadoukhair Z, Belbaraka R, Iraqui M, Boutayeb S, M'rabti H et al. Metastatic ameloblastoma responding to combination chemotherapy: case report and review of the literature. J Med Case Reports. 2011;5:491

[4]. Kim SG, Jang HS. Ameloblastoma: A clinical, radiographic and histopathologic analysis of 71 cases. Oral Surg Oral Med Oral Pathol Oral Radiol Endod. 2001;91:649-53.

[5]. Mendenhall WM, Werning JW, Fernandes R, Malyapa RS, Mendenhall NP. Ameloblastoma. Am J Clin Oncol. 2007;30:645-648.

[6]. Barnes L, Eveson JW, Reichart P, Sidransky D, editors. World health organization classification of tumours: Head and neck tumours. Lyon, France: IARC Press; 2005.

[7]. Gardner DG. Some current concepts on the pathology of ameloblastomas. Oral Surg Oral Med Oral Pathol Oral Radiol Endod. 1996;82(6):660-669.

[8]. Chapelle KAOM, Stoelinga PJW, Wilde PCM, Brouns JJA, Voorsmit RACA. Rational approach to diagnosis and treatment of ameloblastomas and odontogenic keratocysts. Br J Oral Maxillofac Surg. 2004;42:381-390.

[9]. Hong J, et al. Long-term follow up on recurrence of 305 ameloblastoma cases. Int J Oral Maxillofac Surg. 2007;36:283-288.

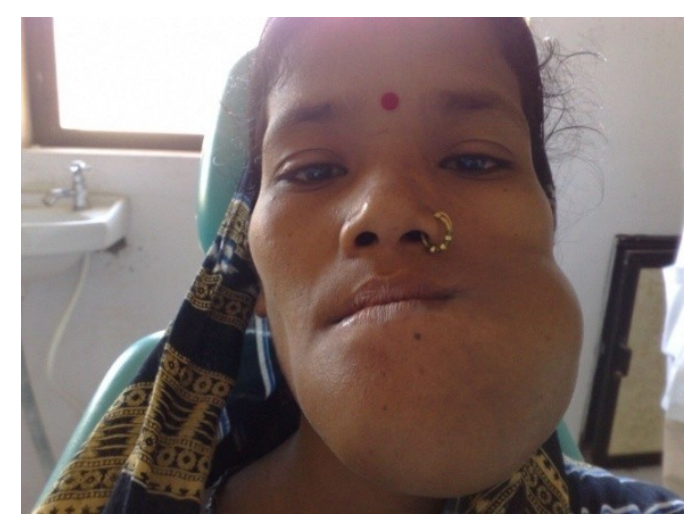

Figure 1: Extraoral presentation.

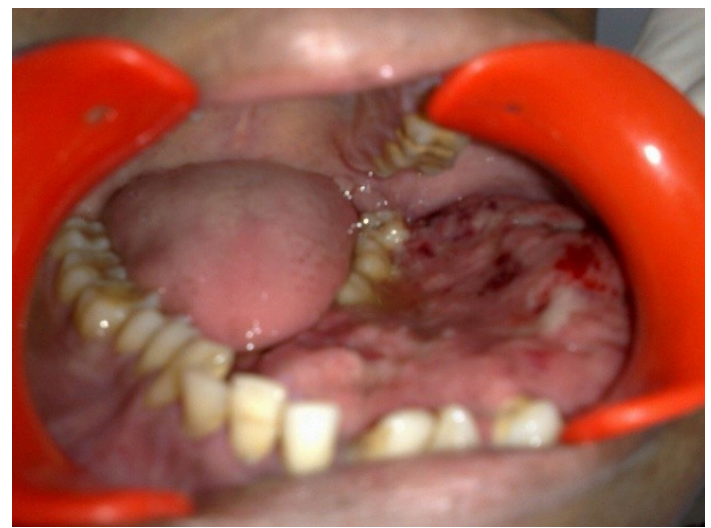

Figure 2: Intraoral presentation. 